\section{ATU-9 AMBULATORY CARE MANAGEMENT OF 70 PATIENTS WITH ACUTE SEVERE UC IN COMPARISON TO 700 INPATIENTS}

${ }^{1}$ Kamal Patel ${ }^{*},{ }^{2}$ Jonathan Segal, ${ }^{3}$ Shaji Sebastian, ${ }^{4}$ Alexandra Kent, ${ }^{2}$ Lucy Hicks, ${ }^{5}$ Shameer Mehta, ${ }^{6}$ Matthew Brookes, ${ }^{7}$ Chris Lamb, ${ }^{8}$ Nicholas Kennedy, ${ }^{9}$ Garerth Walker. ${ }^{1}$ St George's University Hospitals Nhs Foundation Trust, London, UK; ${ }^{2}$ Imperial College London, London, UK; ${ }^{3}$ Hull University Teaching Hospitals NHS Trust, Hull, UK; ${ }^{4}$ King's College Hospital NHS Foundation Trust, London, UK; ${ }^{5}$ University College London Hospitals NHS Foundation Trust, London, UK; ${ }^{6}$ Royal Wolverhampton NHS Trust, Wolverhampton, UK; ${ }^{7}$ Newcastle upon Tyne Hospitals NHS Foundation Trust, Newcastle, UK; ${ }^{8}$ Royal Devon and Exeter NHS Foundation Trust, Exeter, UK; ${ }^{9}$ Torbay and South Devon NHS Foundation Trust, Torbay, UK

\subsection{6/gutjnl-2021-BSG.42}

Introduction Acute severe ulcerative colitis (ASUC) traditionally requires inpatient hospital management for intravenous therapies and/or colectomy. Patients with ASUC can deteriorate rapidly and hence require close monitoring of vital signs correlated with clinical, biochemical and radiological investigations. Traditionally, patients are admitted to hospital to facilitate endoscopic assessment, exclude concomitant infective complications, monitor response to first-line corticosteroid treatment and determine the need for and timing of rescue therapy and/or colectomy. Ambulatory care pathways, which utilise outpatient monitoring and drug delivery, have been shown to deliver safe and effective treatment for conditions

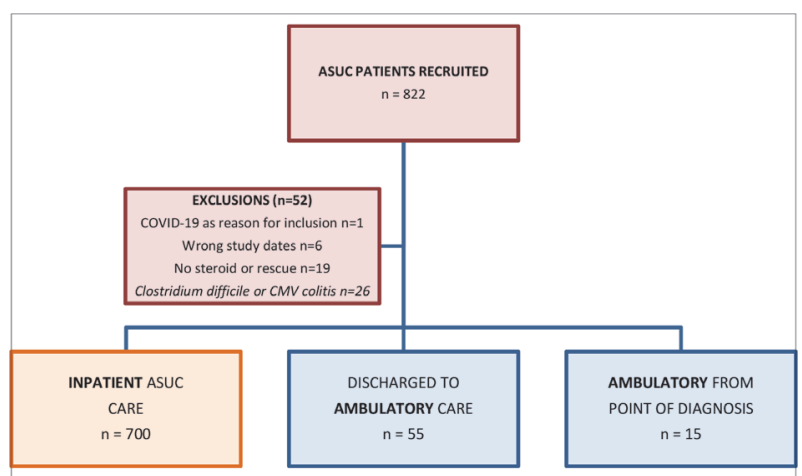

Abstract ATU-9 Figure 1 which have historically mandated hospitalisation e.g. pulmonary embolus. To date there are a paucity of data regarding the use of ambulatory pathways in ASUC cohorts. We used data from PROTECT, a UK multicentre observational COVID19 inflammatory bowel disease (IBD) study, to report the extent, safety and effectiveness of ASUC ambulatory pathways. Methods Adults ( $\geq 18$ years old) meeting Truelove and Witts criteria between 01/01/2019-01/06/2019 and 01/03/2020-30/ 06/2020 were recruited to PROTECT (Abstract ATU9 Figure 1). We utilised demographic, disease phenotype, treatment outcomes and 3-month follow-up data. Primary outcome was rate of rescue therapy and/or colectomy. Secondary outcomes included corticosteroid response, response to rescue therapy, colectomy, mortality and hospital readmission within 3months. We compared outcomes in 3 cohorts: i) patients treated entirely in inpatient setting; ambulatory patients subdivided into ii) patients hospitalised and subsequently discharged to ambulatory care; iii) patients managed as ambulatory from diagnosis.

Results 38\%(23/60) participating hospitals used ambulatory pathways. Of 770 eligible patients, 700(91\%) patients received entirely inpatient care, 55(7\%) patients were discharged to ambulatory pathways and $15(2 \%)$ patients were managed as ambulatory from diagnosis. The rate of rescue therapy and/or colectomy $(49 \%[339 / 696]$ vs $41 \%[22 / 54]$ vs $67 \%[10 / 15]$, respectively, $\mathrm{p}=0.18$ ) (Abstract ATU9 figure 2) and secondary outcomes were similar among all three cohorts. After 3months follow up from the index ASUC diagnosis there was no significant difference in either rate of UC flare, readmission to hospital with UC flare or colectomy between the cohorts.

Conclusions In the largest description of ambulatory ASUC care to date, we report an emerging practice which challenges treatment paradigms. We recommend that patients managed in the ambulatory setting are reviewed by gastroenterologists on a daily basis to monitor clinical parameters and assess for potential complications including venous thromboembolism and biochemical disturbance. Our data suggest ambulatory ASUC treatment may be safe and effective in selected patients but further studies exploring clinical and cost effectiveness as well as patient and physician acceptability are needed.
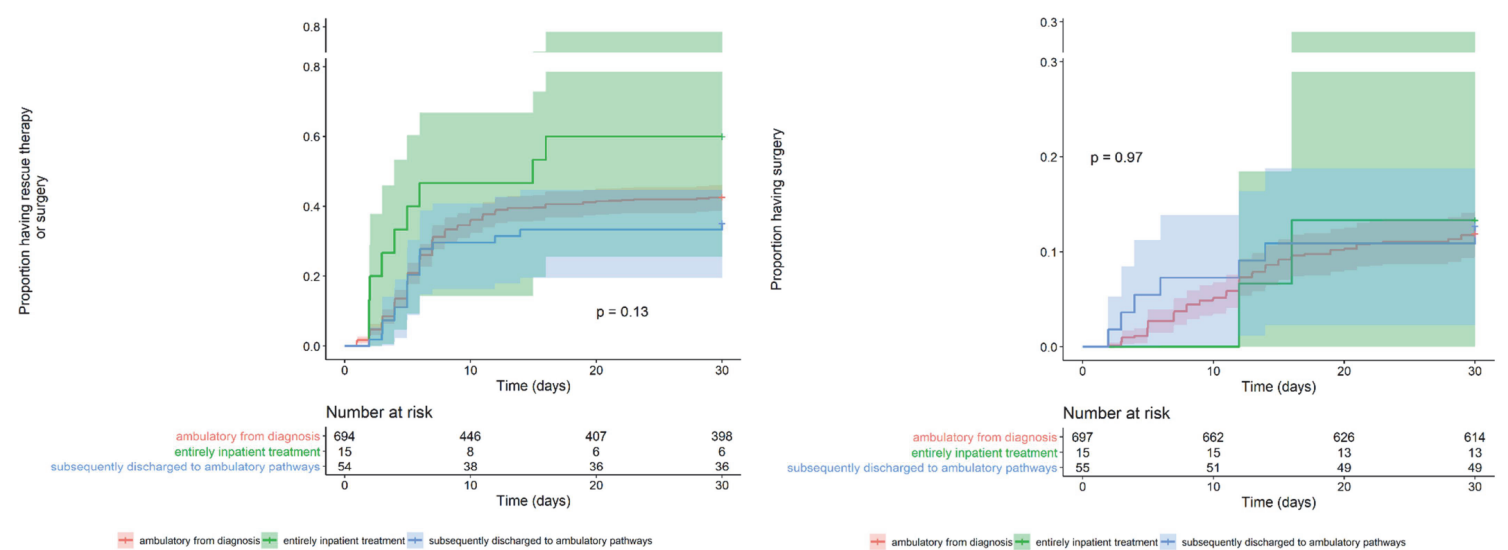

Abstract ATU-9 Figure 2 